\title{
Experimental observation of mitochondrial oxidative damage of liver cells induced by isonicotinic acid hydrazide
}

\author{
GUOYING ZHENG, MANMAN WANG, QI REN, TIESHENG HAN, \\ YUHONG LI, SHUFENG SUN, XUE LI and FUMIN FENG

\begin{abstract}
Key Laboratory of Occupational Health and Safety, School of Public Health, North China
University of Science and Technology, Tangshan, Hebei 063000, P.R. China
\end{abstract}

Received September 22, 2016; Accepted May 11, 2017

DOI: $10.3892 /$ etm.2019.7417

\begin{abstract}
The aim of the present study was to investigate the oxidative damage of liver mitochondria as an adverse effect of the anti-tuberculosis drug isonicotinic acid hydrazide (INH). The human hepatoblastoma cell line (HepG2) was exposed to INH at concentrations of $0,1,2$ or $4 \mathrm{mg} / \mathrm{ml}$ for 24, 48, 72 or $96 \mathrm{~h}$, and the levels of malondialdehyde (MDA), superoxide dismutase (SOD), glutathione peroxidase (GSH-Px) and 8-hydroxy-2-deoxyguanosine $(8-\mathrm{OHdG})$ in mitochondria were detected. Changes in the mitochondrial ultrastructure were observed by electron microscopy. Along with the increase of incubation time and dose of INH, activities of mitochondrial SOD and GSH-Px decreased, MDA and 8-OHdG content increased, and the mitochondrial ultrastructure displayed varying degrees of pathological changes. In conclusion, INH was found to cause liver cell injury by inducing mitochondrial DNA damage.
\end{abstract}

\section{Introduction}

By inhibiting the synthesis of mycobacterium-specific mycolic acid, isonicotinic acid hydrazide (INH) causes the loss of acid resistance and hydrophobicity, followed by proliferation arrest and death of bacteria. It kills bacteria inside and outside of host cells in the breeding and resting state (1). INH is an irreplaceable first-line drug recommended by the World Health Organization as a standard chemotherapy for tuberculosis (TB) (2). However, INH causes changes of liver function and even serious liver injury in certain patients, resulting in the interruption of chemotherapy and TB drug resistance, therefore bringing great difficulties to the control of TB (3). The mechanisms by

Correspondence to: Professor Fumin Feng, Key Laboratory of Occupational Health and Safety, School of Public Health, North China University of Science and Technology, 57 Jianshe Road, Tangshan, Hebei 063000, P.R. China

E-mail:fm_feng@sina.com

Key words: isonicotinic acid hydrazide, human hepatoblastoma, HepG2 cells, oxidative damage, mitochondrial DNA which anti-TB drugs induce liver toxicity have remained to be fully elucidated (4), making it challenging to develop targeted liver-protective therapies. Therefore, there is an urgent need to explore the mechanisms of anti-TB drug-induced liver injury. The involvement of mitochondria in the pathogenesis of anti-TB drug-induced hepatotoxicity (ADIH) has gained much attention in recent years. Mitochondria are important organelles within eukaryotic cells, which generate adenosine triphosphate (ATP) through an oxidative phosphorylation reaction with electron transfer for the body (5). Mitochondria have further important functions, including the generation of reactive oxygen species, regulation of the cell's redox potential, signal transduction, apoptosis and regulation of gene expression. Therefore, mitochondria have significant roles in cell growth, metabolism and disease development (6).

Mammalian liver cells contain abundant mitochondria. As the liver has a major role in the metabolization of INH, the liver mitochondria are exposed to the toxic metabolites of INH and are easily damaged (7). As mitochondrial DNA (mtDNA) is bare, lacking the protection of histones and DNA-binding proteins as well as effective repair mechanisms, they are vulnerable to damage $(8,9)$. Numerous studies have identified that mtDNA is closely associated with oxidative damage and mitochondrial myopathy, neurodegenerative diseases and aging (10-12). The present study examined the effect of INH on mitochondrial anti-oxidant enzymes and mitochondrial DNA, aiming to provide clues regarding the etiology of drug-induced liver injury.

\section{Materials and methods}

Instruments and reagents. Dulbecco's modified Eagle's medium (DMEM), fetal bovine serum and $0.25 \%$ trypsin were obtained from Gibco (Thermo Fisher Scientific, Inc., Waltham, MA, USA). A Mitochondrial Extraction kit (CA: SM0020) was purchased from Beijing Solarbio Co., Ltd. (Beijing, China). MDA (CA: A003-2), SOD (CA: A001-3) and GSH-PX (CA: A005) assay kit were from Nanjing Jiancheng Bioengineering Institute (Nanjing, China). 8-OHdG ELISA kit (CA: ab201734; Abcam, Cambridge, MA, USA). INH injection was obtained from Tianjin Kingyork Group Co., Ltd (Tianjin, China). HepG2 cells were supplied by Obio Technology Co., Ltd. (Shanghai, China). The plate reader 
was from Molecular Devices (Sunnyvale, California USA; SpectraMax M5).

Cell culture and treatments. HepG2 cells were routinely cultured in DMEM complete culture medium containing $10 \%$ fetal bovine serum, with culture conditions of $37^{\circ} \mathrm{C}$ and $5 \% \mathrm{CO}_{2}$. Based on the INZ concentrations added (13), cells were divided into control group, low-dose group $(1 \mathrm{mg} / \mathrm{ml})$, medium-dose group $(2 \mathrm{mg} / \mathrm{ml})$ and high-dose group $(4 \mathrm{mg} / \mathrm{ml})$, with a treatment time of 24, 48, 72 or $96 \mathrm{~h}$. Each group had six replicates.

Mitochondrial protein quantification. After 48 or $96 \mathrm{~h}$ of treatment, cells were collected by centrifugation at $800 \mathrm{x} \mathrm{g}$ for $5 \mathrm{~min}$. The protein concentration of each extracted mitochondrial sample was measured strictly following the instructions of the measurement kit. The absorbance was measured at $562 \mathrm{~nm}$ on a microplate reader, and the protein concentration of each sample was calculated according to a standard curve. The extracted mitochondria were used immediately or stored at $-70^{\circ} \mathrm{C}$ in storage buffer for later detection.

Determination of malondialdehyde (MDA) and anti-oxidant enzymes in mitochondria. MDA and the enzymatic activity of superoxide dismutase (SOD) and glutathione peroxidase (GSH-Px) were measured using the above mentioned commercial kits following the manufacturer's instructions.

Determination of mitochondrial 8-hydroxy-2-deoxyguanosine (8-OHdG) content. Mitochondrial 8-OHdG was measured by 8-OHdG ELISA kit following the instructions of the manufacturer. Absorbance values were measured on a microplate reader.

Ultrastructural observation. Following treatment, cells were collected by centrifugation at $800 \mathrm{xg}$ for $5 \mathrm{~min}$ at $4^{\circ} \mathrm{C}$. Cells were fixed in $2.5 \%$ glutaraldehyde $(\mathrm{v} / \mathrm{v})$ immediately on ice for $2 \mathrm{~h}$, post-fixed for $1 \mathrm{~h}$ in $1 \%(\mathrm{v} / \mathrm{v})$ osmium tetroxide, dehydrated in acetone and embedded in Epon 812 (02635-AB; SPI Supplies; West Chester, Pennsylvania, USA). From appropriate locations, ultrathin sections $(50 \mathrm{~nm})$ were obtained and the sections were stained with $3 \%$ uranyl acetate and lead citrate, followed by ultrastructural observation under an electron microscope (HitachiH-7650; Hitachi, Ltd., Tokyo, Japan) operated at $80 \mathrm{KV}$.

Statistical analysis. Values are expressed as the mean \pm standard error. Differences among groups were tested by one-way analysis of variance and least-significant differences post-hoc test using SPSS 13 software (SPSS, Inc., Chicago, IL, USA). $\mathrm{P}<0.05$ was considered to indicate a statistically significant difference.

\section{Results}

Mitochondrial anti-oxidant enzyme and MDA measurements SOD activity. SOD activity in the different groups is displayed in Fig. 1. Statistical analysis showed that SOD activity in the high-dose group at $72 \mathrm{~h}$ was significantly lower $(\mathrm{P}<0.001)$ than that in the control group, SOD activity in the high-dose group at $96 \mathrm{~h}$ was significantly lower than that in all other three groups (compared with control, low-dose and medium-dose groups; $\mathrm{P}<0.001, \mathrm{P}<0.001$ and $\mathrm{P}=0.015)$. In addition, the activity of SOD in the medium-dose group at $96 \mathrm{~h}$ was significantly lower $(\mathrm{P}=0.005)$ than that in the control group. Analysis of variance showed that the concentration $(\mathrm{F}=6.099, \mathrm{P}=0.001)$, time $(\mathrm{F}=11.088, \mathrm{P}<0.001)$ and the interaction between concentration and time $(\mathrm{F}=2.135, \mathrm{P}=0.036)$ were significant, which demonstrated that time and concentration both have an effect on SOD activity. These findings suggest that SOD activity was reduced along with the increase of the INH concentration and incubation time.

GSH-Px activity. The activity of GSH-Px in the different experimental groups is presented in Fig. 2. Statistical analysis indicated that GSH-Px activity in the high-dose group at $72 \mathrm{~h}$ was significantly lower than that in the control group $(\mathrm{P}<0.001)$ and low dose group $(\mathrm{P}=0.015)$. GSH-Px activity in the high-dose group at $96 \mathrm{~h}$ was significantly lower than that in the all other three groups (compared with control, low-dose and medium-dose groups; $\mathrm{P}<0.001, \mathrm{P}<0.001, \mathrm{P}=0.048$ ). Furthermore, GSH-Px activity in the medium-dose group at $96 \mathrm{~h}$ was significantly lower $(\mathrm{P}=0.001)$ than that in the control group. Analysis of variance revealed that the concentration $(\mathrm{F}=10.233, \mathrm{P}<0.001)$, time $(\mathrm{F}=11.448, \mathrm{P}<0.001)$ and the interaction between concentration and time $(\mathrm{F}=2.077, \mathrm{P}=0.041)$ were significant, which demonstrated that time and concentration both have effect on GSH-PX activity. These results demonstrated that GSH-PX activity was reduced along with the increase of INH concentration and incubation time.

MDA concentration. The MDA concentration in the different experimental groups is displayed in Fig. 3. Statistical analysis suggested that MDA level in the high-dose group at $72 \mathrm{~h}$ was significantly higher than that in the control group $(\mathrm{P}=0.005)$ and low-dose group $(\mathrm{P}=0.048)$. MDA level in the high-dose group at $96 \mathrm{~h}$ was significantly higher than that in all other three groups (compared with control, low-dose and medium-dose groups; $\mathrm{P}<0.001, \mathrm{P}<0.001, \mathrm{P}<0.001)$. In addition, $\mathrm{MDA}$ activity in the medium-dose group at $96 \mathrm{~h}$ was significantly higher $(\mathrm{P}=0.006)$ than that in the control group. Analysis of variance showed that the concentration $(\mathrm{F}=18.126, \mathrm{P}<0.001)$, time $(\mathrm{F}=12.110, \mathrm{P}<0.001)$ and the interaction between concentration and time $(\mathrm{F}=3.724, \mathrm{P}=0.001)$ was significant, which demonstrated that time and concentration both have effects on MDA level. The MDA content increased along with the increase of INH concentration and incubation time.

Mitochondrial 8-OHdG levels. The levels of 8-OHdG are presented in Fig. 4. Statistical analysis indicated that the levels of $8-\mathrm{OHdG}$ in the high-dose group at $72 \mathrm{~h}$ (compared with control, low-dose and medium-dose groups; $\mathrm{P}<0.001$, $\mathrm{P}<0.001, \mathrm{P}=0.002)$ and $96 \mathrm{~h}$ (compared with control, low-dose and medium-dose groups; $\mathrm{P}<0.001, \mathrm{P}<0.001$ and $\mathrm{P}<0.001$ ) were significantly higher. Furthermore, $8-\mathrm{OHdG}$ activity in the medium-dose group at $72 \mathrm{~h}$ was significantly higher than that in the control group $(\mathrm{P}=0.016)$, and the medium-dose group at $96 \mathrm{~h}$ was significantly higher than that in the control group $(\mathrm{P}<0.001)$ and low-dose group $(\mathrm{P}<0.001)$. Analysis of variance shows that the concentration $(\mathrm{F}=68.323, \mathrm{P}<0.001)$, 


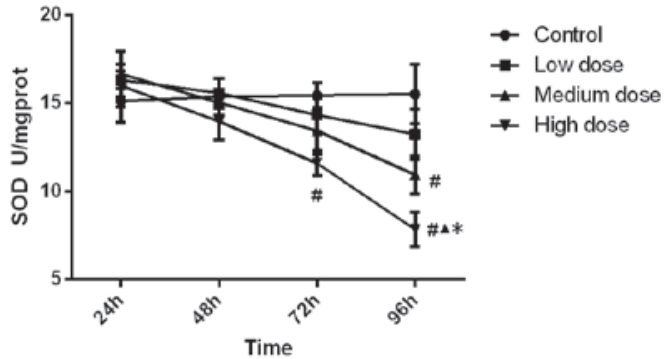

Figure 1. SOD activity in the experimental groups. SOD activity was reduced along with the increase of isonicotinic acid hydrazide concentration and incubation time, ${ }^{\text {"}} \mathrm{P}<0.05$ vs. Control; ${ }^{\boldsymbol{\Delta}} \mathrm{P}<0.05$ vs. Low-dose; ${ }^{*} \mathrm{P}<0.05$ vs. medium dose. SOD, superoxide dismutase; prot, protein.

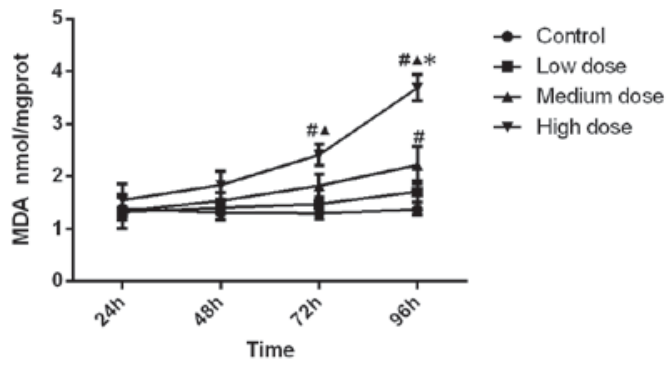

Figure 3. MDA content in the experimental groups. The MDA content increased along with the increase of isonicotinic acid hydrazide concentration and incubation time. ${ }^{*} \mathrm{P}<0.05$ vs. Control; ${ }^{\boldsymbol{\Delta}} \mathrm{P}<0.05$ vs. Low-dose, ${ }^{*} \mathrm{P}<0.05$ vs. medium dose. MDA, malondialdehyde; prot, protein.

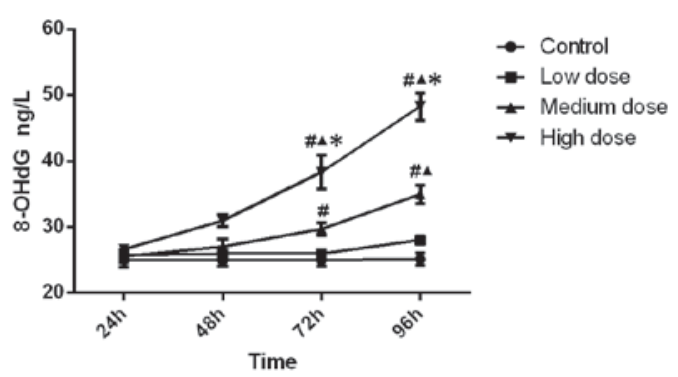

Figure 4. 8-OHdG content in the experimental groups. The mitochondrial 8 -OHdG content increased along with the increase of isonicotinic acid hydrazide dose and incubation time. ${ }^{\#} \mathrm{P}<0.05$ vs. Control; ${ }^{\mathbf{\Delta}} \mathrm{P}<0.05$ vs. Low-dose; ${ }^{*} \mathrm{P}<0.05$ vs. medium dose. 8-OHdG, 8-hydroxy-2-deoxyguanosine.

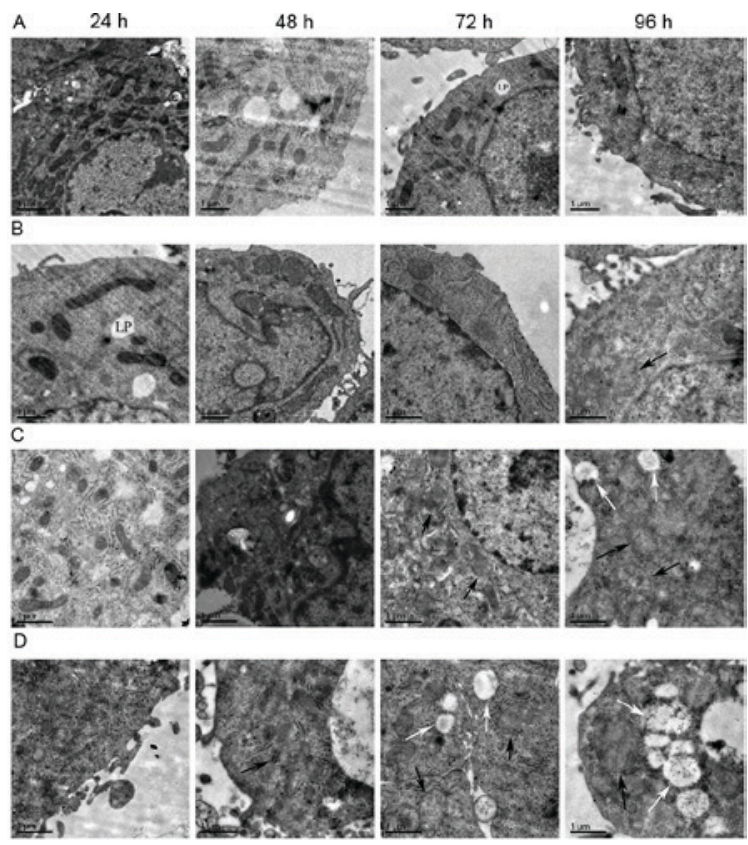

As the major organelles for energy production $(14,15)$, mitochondria are the main cellular site of the Krebs cycle, electron transport and oxidative phosphorylation. Therefore, they are the main source of reactive oxygen radicals and the primary target of oxidative damage $(16,17)$. Under normal circumstances, the generation and clearance of oxygen radicals is in a dynamic equilibrium state. However, mitochondrial oxidative stress occurs if this balance is disrupted, resulting in mitochondrial damage (18).

time $(\mathrm{F}=38.135, \mathrm{P}<0.001)$ and the interaction between concentration and time $(\mathrm{F}=12.929, \mathrm{P}<0.001)$ was significant, which demonstrated that time and concentration both have effects on 8-OHdG level. The results indicated that the mitochondrial 8-OHdG content increased along with the increase of INH dose and incubation time.

Effect of isoniazid on mitochondrial morphology of HepG2 cells. As displayed in Fig. 5, swelling of mitochondria of the HepG2 cells was observed in the low-dose 96-h group, medium-dose 72-h group and high-dose 48-h group. In the medium-dose 96-h group, high-dose $72 \mathrm{~h}$ group and high-dose 6-h group, mitochondrial swelling and vacuoles within certain mitochondria were visible. The mitochondrial vacuolation was changes in mitochondrial morphology in the remaining groups.

\section{Discussion}


SOD and GSH-Px are important members of the anti-oxidant defense system. SOD is the first line of defense against oxygen free radicals and catalyzes the conversion of superoxide anion radicals into $\mathrm{H}_{2} \mathrm{O}_{2}$, which is further cleared by GSH-Px. MDA is a product of lipid peroxidation, and reflects the level of free radical production and oxidative damage (19). The present study demonstrated that the MDA content increased along with the increase of the INH concentration and incubation time, indicating that INH dose- and time-dependently induced mitochondrial oxidative damage. In consistency with that, SOD and GSH-PX activity were reduced along with the increase of INH concentration and incubation time. These results suggested that INH impairs the ability of these enzymes to scavenge oxygen radicals, causing lipid peroxidation and mitochondrial damage.

Compared to nuclear DNA, mtDNA is more sensitive to oxidative damage. mtDNA is a bare DNA lacking protein protection (20), and its ability to repair damage is therefore poor (21). mtDNA is connected with the mitochondrial inner membrane, which is full of lipids, rendering mtDNA sensitive to damage. $8-\mathrm{OHdG}$ is the most commonly used biomarker for mtDNA damage (22). The results of the present study demonstrated that the mitochondrial 8-OHdG content increased along with the increase of the INH dose and incubation time, suggesting that INH causes mtDNA damage. Since mtDNA has no intron sequence except for a short region of replication and transcription, any damage to mtDNA may significantly affect mitochondrial function. Studies have suggested that the vast majority of mtDNA mutations may affect the respiratory chain function and further aggravate the decrease of the activity of respiratory chain complexes, resulting in increased oxygen radical levels and reduction in ATP synthesis. As the oxidation products continue to accumulate, mtDNA loss, mutation or large fragmentation occurs, leading to the loss of its important function to various extents $(23,24)$. Furthermore, mtDNA fragment deletion was reported to be closely associated with the loss of direct repeat sequences during the process of mtDNA replication and damage through oxygen free radicals (25). Mitochondrial DNA fragment deletion may cause the lack of genes encoding transfer RNAs and proteins involved in the mitochondrial respiratory chain, resulting in the dysfunction of the electronic chain, oxidative phosphorylation and electron leakage. This may also cause immature electron breathing and increase the generation of oxygen free radicals, which further promotes mitochondrial damage.

Projection electron microscopy results of the cell mitochondrial ultrastructure demonstrated that mitochondria of INH-treated HepG2 cells were swollen in the low-dose 96-h group, medium-dose 72-h group and high-dose 48-h group. Mitochondria swelling and vacuoles were found in certain mitochondria in the medium-dose 96-h group, high-dose $72 \mathrm{~h}$ group and high-dose $96-\mathrm{h}$ group. These results confirmed at the ultrastructural level that the mitochondrial damage increased along with the increase of INH dose and incubation time.

A previous study on the toxic effects of INH mainly focused on apoptosis of HepG2 cells (13). The underlying mechanisms have remained to be fully elucidated. The present study investigated the effects of INH on mitochondrial damage in HepG2 cells and the underlying molecular mechanisms. It was revealed that INH-induced hepatic cell apoptosis and liver injury are likely caused by mtDNA damage.
The limitations of the present study investigation include the lack of an in vivo study and further studies using animal models are required to confirm the conclusions. However, to the best of our knowledge, the present study was the first to provided direct evidence for INH-induced mitochondrial damage and may enhance the current understanding on the mechanisms of ADIH. The present study may provide a theoretical basis for the development of novel therapeutic strategies for ADIH.

\section{Acknowledgements}

The authors would like to acknowledge the support of the present study by Tangshan Science and Technology Bureau (grant no. 08150201A-1-8).

\section{References}

1. Zhang Z, Song L, He L, Gao L, Shi Z, Tian X, Zhang G and Feng F: Experimental study on the expression of nuclear factor erythroid 2-related factor-2 and superoxide dismutase during the isoniazid-induced liver injury. Chin J Infect Dis 32: 80-84, 2014.

2. Boelsterli UA and Lee KK: Mechanisms of isoniazid-induced idiosyncratic liver injury: Emerging role of mitochondrial stress. J Gastroenterol Hepatol 29: 678-687, 2014.

3. Corbett EL, Watt CJ, Walker N, Maher D, Williams BG, Raviglione MC and Dye C: The growing burden of tuberculosis: Global trends and interactions with the HIV epidemic. Arch Intern Med 163: 1009-1021, 2003.

4. Ramappa V and Aithal GP: Hepatotoxicity related to anti-tuberculosisdrugs: Mechanisms and management. J Clin Exp Hepatol 3: 37-49, 2013.

5. Misu H, Takamura T, Matsuzawa N, Shimizu A, Ota T, Sakurai M, Ando H, Arai K, Yamashita T, Honda M, et al: Genes involved in oxidative phosphorylation are coordinately upregulated with fasting hyperglycaemia in livers of patients with type 2 diabetes. Diabetologia 50: 268-277, 2007.

6. Srinivasula SM, Hegde R, Saleh A, Datta P, Shiozaki E, Chai J, Lee RA, Robbins PD, Fernandes-Alnemri T, Shi Y and Alnemri ES: A conserved XIAP-interaction motif in caspase-9 and Smac/DIABLO regulates caspase activity and apoptosis. Nature 410: 112-116, 2001.

7. Labbe G, Pessayre D and Fromenty B: Drug-induced liver injury through mitochondrial dysfunction: Mechanisms and detection during preclinical safety studies. Fundam Clin. Pharmacol 22: 335-353, 2008.

8. Balaban RS, Nemoto S and Finkel T: Mitochondria, oxidants, and aging. Cell 120: 483-495, 2005.

9. Chien KR and Karsenty G: Longevity and lineagea: Toward the integrative biology of degenerative diseases in heart, muscle, and bone. Cel1 120: 533-544, 2005.

10. Tanahashi $\mathrm{C}$, Nakayama A, Yoshida M, Ito M, Mori $\mathrm{N}$ and Hashizume Y: MELAS with the mitochondrial DNA 3243 point mutation: A neuropathological study. Acta Neuropathol 99: 31-38, 2000.

11. Barthélémy C, Ogier de Baulny H, Diaz J, Cheval MA, Frachon P, Romero N, Goutieres F, Fardeau M and Lombès A: Late-onset mitochondrial DNA depletion: DNA copy number, multiple deletions and compensation. Ann Neurol 49: 607-617, 2001.

12. Leonard JV and Schapira AH: Mitochondrial respiratory chain disorders I: Mitochondrial DNA defects. Lancet 355: 299-304, 2000.

13. Yang B, Zhang L, Liu JW and An Y: Study on the isoniazid induced cellular damage and the expressions of Fas/Fas ligand of HepG2. Chin J Infect Dis 30: 402-406, 2012.

14. Yang $\mathrm{L}$ and Yu T: Prolonged donor heart preservation with pinacidil: The role of mitochondria and the mitochondrial adenosinetriphosphate-sensitive potassium channel. J Thorac Cardiovasc Surg 139: 1057-1063, 2010.

15. Simon DK, Pankratz N, Kissell DK, Pauciulo MW, Halter CA, Rudolph A, Pfeiffer RF, Nichols WC and Foroud T; Parkinson Study Group-PROGENI Investigators: Maternal inheritance and mitochondrial DNA variants in familial Parkinson's disease. BMC Med Genet 11: 53, 2010. 
16. Peng TI and Jou MJ: Mitochondrial swelling and generation of reactive oxygen species induced by photoirradiation are heterogeneously distributed. Ann N Y Acad Sci 1011: 112-122, 2004.

17. Choksi KB, Boylston WH, Rabek JP, Widger WR and Papaconstantinou J: Oxidatively damaged proteins of heart mitochondrial electron transport complexes. Biochim Biophys Acta 1688: 95-101, 2004.

18. Cortes-Rojo C and Rodríguez-Orozco AR: Importance of oxidative damage on the electon transport chain for the rational use of mitochondria-targeted antioxidants. Mini Rev Med Chem 11: 625-632, 2011.

19. Fattman CL, Schaefer LM and Oury TD: Extracellular superoxide dismutase in biology and medicine. Free Radie Biol Med 35: 236-256, 2003

20. Clayton DA: Replication of animal mitochondrial DNA. Cell 28: 693-705, 1982

21. Bandy B and Davison AJ: Mitochondrial mutations may increase oxidative stress: Implications for carcinogenesis and aging? Free Radic Biol Med 8: 523-539, 1990.
22. Cooke MS, Evans MD, Burd RM, Patel K, Barnard A, Lunec J and Hutchinson PE: Induction and excretion of ultraviolet-induced 8-OXO-2'-deoxyguanosine and thymine dimers in vivo: Implications for PUVA. J Invest DermatoI 116: 281-285, 2001 .

23. Ingman M and Gyllensten U: Analysis of the complete human mtDNA genome: Methodology and inferences for human evolution. J Hered 92: 454-461, 2001.

24. Tanaka M, Kovalenko SA, Gong JS, Borgeld HJ, Katsumata K, Hayakawa M, Yoneda M and Ozawa T: Accumulation of deletions and point mutations in mitochondrial genome in degenerative diseases. Ann N Y Acad Sci 786: 102-111, 1996.

25. Berneburg M, Grether-Beck S, Kürten V, Ruzicka T, Briviba K, Sies H and Krutmann J: Singlet oxygen mediates the UVA-induced generation of the photoaging-associated mitochondrial common deletion. J Biol Chem 274: 15345-15395, 1999. 